Abstract P863 Table 1 DLT, TRAE, and ORR in KEYNOTE-022 parts 4 and 5

\begin{tabular}{|c|c|c|c|c|c|}
\hline $\mathrm{n}(\%)$ & $\begin{array}{l}\mathrm{cDL1} \\
\mathrm{n}=5\end{array}$ & $\begin{array}{l}\mathrm{cDL2a} \\
\mathrm{n}=16\end{array}$ & $\begin{array}{l}\mathrm{cDL2b} \\
\mathrm{n}=3\end{array}$ & $\begin{array}{l}\mathrm{iDL1} \\
\mathrm{n}=3\end{array}$ & $\begin{array}{l}\mathrm{iDL2} \\
\mathrm{n}=15\end{array}$ \\
\hline DLT & $n=0$ & $\begin{array}{l}\text { Grade } 2 \text { diarrhea, } n=1 ; \text { grade } 1 \\
\text { thrombocytopenia, } n=1 ; \text { grade } \\
2 \text { dermatitis acneiform, } n=1 ; \\
\text { grade } 2 \text { rash, } n=1\end{array}$ & $\begin{array}{l}\text { Grade } 4 \\
\text { thrombocytopenia, } n=1 \text {; } \\
\text { grade } 2 \text { mucosal } \\
\text { inflammation, } n=1\end{array}$ & $\mathrm{n}=0$ & $\begin{array}{l}\text { Grade } 2 \text { rash, } n=2 ; \\
\text { grade } 3 \text { aseptic } \\
\text { meningitis, } n=1 ; \\
\text { grade } 3 \text { pneumonitis, } \\
n=1\end{array}$ \\
\hline $\begin{array}{l}\text { Dose selected } \\
\text { for confirmation } \\
\text { in part } 5\end{array}$ & No & Yes & No & No & Yes \\
\hline $\begin{array}{l}\text { Any-grade } \\
\text { TRAE }\end{array}$ & $\begin{array}{l}5 \\
(100.0)\end{array}$ & $\begin{array}{l}13 \\
(81.3)\end{array}$ & $\begin{array}{l}3 \\
(100.0)\end{array}$ & $\begin{array}{l}3 \\
(100.0)\end{array}$ & $\begin{array}{l}15 \\
(100.0)\end{array}$ \\
\hline $\begin{array}{l}\text { Grade } 3-5 \\
\text { TRAE }\end{array}$ & $\begin{array}{l}5 \\
(100.0)\end{array}$ & $\begin{array}{l}5 \\
(31.3)\end{array}$ & $\begin{array}{l}2 \\
(66.7)\end{array}$ & $\begin{array}{l}0 \\
(0)\end{array}$ & $\begin{array}{l}7 \\
(46.7)\end{array}$ \\
\hline ORR & $\begin{array}{l}2 \\
(40.0)\end{array}$ & $\begin{array}{l}0 \\
(0)\end{array}$ & $\begin{array}{l}0 \\
(0)\end{array}$ & $\begin{array}{l}1 \\
(33.3)\end{array}$ & $\begin{array}{l}4 \\
(26.7)\end{array}$ \\
\hline CR & $\begin{array}{l}1 \\
(20.0)\end{array}$ & $\begin{array}{l}0 \\
(0)\end{array}$ & $\begin{array}{l}0 \\
(0)\end{array}$ & $\begin{array}{l}0 \\
(0)\end{array}$ & $\begin{array}{l}0 \\
(0)\end{array}$ \\
\hline PR & $\begin{array}{l}1 \\
(20.0)\end{array}$ & $\begin{array}{l}0 \\
(0)\end{array}$ & $\begin{array}{l}0 \\
(0)\end{array}$ & $\begin{array}{l}1 \\
(33.3)\end{array}$ & $\begin{array}{l}4 \\
(26.7)\end{array}$ \\
\hline
\end{tabular}

cDL1: 4 weeks of trametinib $1.5 \mathrm{mg}$ QD run-in, concurrent dosing of pembrolizumab $200 \mathrm{mg}$ Q3W and trametinib $1.5 \mathrm{mg}$ QD starting at week 5 .

cDL2a: 2 weeks of trametinib $1.5 \mathrm{mg}$ QD run-in, concurrent dosing of pembrolizumab $200 \mathrm{mg}$ Q3W and trametinib $1.5 \mathrm{mg} \mathrm{QD}$ starting at week 3 .

cDL2b: 4 weeks of trametinib $2 \mathrm{mg}$ QD run-in, concurrent dosing of pembrolizumab $200 \mathrm{mg}$ Q $3 \mathrm{~W}$ and trametinib $2 \mathrm{mg}$ QD starting at week 5.

iDL1: 2 weeks of trametinib $1.5 \mathrm{mg}$ QD run-in, pembrolizumab $200 \mathrm{mg}$ Q3W dosing starting at week 3 with intermittent 1 week off trametinib, 2 weeks on trametinib $1.5 \mathrm{mg} \mathrm{QD}$.

iDL2: 2 weeks of trametinib $2 \mathrm{mg}$ QD run-in, pembrolizumab $200 \mathrm{mg}$ Q3W dosing starting at week 3 with intermittent 1 week off trametinib, 2 weeks on trametinib $2 \mathrm{mg}$ QD.

DLT, dose-limiting toxicity; TRAE, treatment-related adverse event; ORR, objective response rate.

pembrolizumab+trametinib. Safety was analyzed for all patients who received $\geq 1$ dose of study drug; patients treated during the trametinib run-in who discontinued study before receiving pembrolizumab were included; patients who did not complete trametinib run-in or receive $\geq 66 \%$ of planned doses during the 6-week dose-limiting toxicity (DLT) evaluable period were not included for DLT evaluation. AEs were graded per NCI CTCAE v4.

Results Of 42 enrolled patients, most were female (61.9\%); median age was 55.0 years; $57.1 \%$ had received $\geq 2$ prior lines of therapy. At database cutoff (June 26, 2019), median follow-up was 9.0 months (range, 1.4-25.6 months). Of 38 DLT-evaluable patients, 10 had DLTs (table 1). Dosing regimens were selected for confirmation in part 5 based on safety data. Any-grade TRAEs occurred in 39 (92.9\%) patients; grade 3-4 TRAEs occurred in 19 (45.2\%), none were grade 5 . TRAEs led to discontinuation in $8(19.0 \%)$ patients. Immunemediated AEs occurred in $12(28.6 \%)$ patients, most commonly severe skin reactions $(n=6 ; 14.3 \%)$, pneumonitis $(n=3$; 7.1\%), hypothyroidism ( $n=2 ; 4.8 \%)$. The MTD of concurrent pembrolizumab+trametinib was pembrolizumab $200 \mathrm{mg}$ Q3W plus trametinib $1.5 \mathrm{mg}$ with 2 weeks of trametinib run-in (ORR, 0/16;0\%) and the MTD of intermittent pembrolizumab+trametinib was pembrolizumab $200 \mathrm{mg}$ Q3W plus trametinib $2 \mathrm{mg}$ with 2 weeks of run-in (ORR, 4/15; 26.7\%).

Conclusions Both concurrent or intermittent pembrolizumab +trametinib dosing were feasible and the combination showed antitumor activity in patients with advanced solid tumors or advanced BRAF wild-type melanoma.

\section{P864 COMBINATION OF FECAL MICROBIOTA TRANSPLANTATION FROM HEALTHY DONORS WITH ANTI-PD1 IMMUNOTHERAPY IN TREATMENT-NAÏVE ADVANCED OR METASTATIC MELANOMA PATIENTS}

${ }^{1}$ Saman Maleki*, ${ }^{1}$ John Lenehan, ${ }^{2}$ Jeremy Burton, ${ }^{1}$ Michael Silverman, ${ }^{3}$ Seema Nair Parvathy, ${ }^{1}$ Mikal El-Hajjar, 'Mithunah Krishnamoorthy. ${ }^{1}$ Western University, London, Canada; ${ }^{2}$ Lawson Health Research Institute, London, Canada; ${ }^{3}$ St. Joseph's Health Care, London, Canada

\subsection{6/LBA2019.17}

Background Checkpoint inhibitors have changed the outcomes for patients with advanced melanoma. However, many patients still show primary resistance to single-agent therapy. Recently, the role of the gut microbiome in influencing antitumor immunity has been established. Currently, various methods of modifying the gut microbiome of cancer patients are being explored. We report the initial safety results of the first two patients treated on a phase I study combining Fecal Microbiota Transplantation (FMT) with single-agent anti-PD1 in treatment-naïve patients with advanced melanoma.

Methods Two healthy donors were selected through our screening process and approximately 100 grams of fresh stool was processed and prepared for FMT as per our standardized protocol. FMT recipients were melanoma patients with unresectable or metastatic disease who were treatment naïve for their advanced disease. Bowel preparation was completed the day prior and FMT was performed using oral administration of approximately 40 capsules. Anti-PD1 was started at least 1 week after FMT to allow for microbiome engraftment. Blood 
and stool were analyzed at baseline (pre-FMT), before immunotherapy, and three weeks after it.

Results Patient 1 was diagnosed with recurrent melanoma of the lower limb with multiple in-transit lesions refractory to control with surgery and a single intralesional injection of IL2. Patient received stool from Donor 1 and did not experience any adverse effects from FMT. At the time of treatment \#4, a solitary large cutaneous lesion stabilized but the patient experienced grade 1 diarrhoea, grade 2 nausea, and grade 2 fatigue, and grade 2 depression (NCI-CTCAE v5.0). Patient 2 was diagnosed with recurrent melanoma of the parotid gland with metastatic lesions in the lungs. Patient 2 received stool from Donor 2 and experienced only grade 1 flatus from FMT. At the time of treatment \#3, the patient experienced grade 1 constipation. Both patients had a vigorous immune response to FMT measured by changes in the immune subpopulations in peripheral blood one week after FMT, including an increase in CD28 + CD8 + T cells and a decrease in PDL1 + CD3- cells. Following anti-PD1 therapy, both patients had an increase in $\mathrm{CD} 39+\mathrm{CD} 8+\mathrm{T}$ cell population. The PD1+ $\mathrm{CD} 38+\mathrm{CD} 8+$ dysfunctional $\mathrm{T}$ cell levels decreased in both patients post-FMT and anti-PD1 therapy.

Conclusions FMT combined with anti-PD1 therapy in patients with advanced melanoma appears to be safe. A measurable immune response was observed one week after FMT in both patients. One patient experienced several grade 2 toxicities with stabilization of a large cutaneous lesion.

Acknowledgements This study is funded by a grant from The Lotte \& John Hecht Memorial Foundation and a grant from The Medical Oncology Research Funds (MORF) from Western University.

Trial Registration NCT03772899

Ethics Approval The study was approved by Western University Institutution's Ethics Board, approval number 113131, date of approval March 15, 2019.

\section{P865 SAFETY \& EFFICACY OF LIFILEUCEL (LN-144) TUMOR INFILTRATING LYMPHOCYTE THERAPY IN METASTATIC MELANOMA PATIENTS AFTER PROGRESSION ON MULTIPLE THERAPIES - INDEPENDENT REVIEW COMMITTEE DATA UPDATE}

${ }^{1}$ Amod Sarnaik, ${ }^{1}$ Nikhil Khushalani, ${ }^{2}$ Jason Chesney, ${ }^{3}$ Harriet Kluger, ${ }^{4}$ Brendan Curti, ${ }^{5}$ Karl Lewis, ${ }^{5}$ Theresa Medina, ${ }^{6}$ Sajeve Thomas, ${ }^{7}$ Anna Pavlick, ${ }^{8}$ Eric Whitman, ${ }^{9}$ Salvador Algarra, ${ }^{10}$ Pippa Corrie, ${ }^{11}$ Omid Hamid, ${ }^{12}$ Jose Lutzky, ${ }^{13}$ Judit Olah, ${ }^{7}$ Jeffrey Weber, ${ }^{14}$ James Larkin, ${ }^{15}$ Wen Shi, ${ }^{15}$ Kelly DiTrapani, ${ }^{15}$ Harry Qin, ${ }^{15}$ Mariam Mirgoli, ${ }^{15}$ Renee Wu, ${ }^{15}$ Toshimi Takamura, ${ }^{15}$ Maria Fardis, ${ }^{16}$ John Kirkwood*. ${ }^{1} \mathrm{H}$. Lee Moffitt Cancer Center, Tampa, FL, USA; ${ }^{2}$ James Graham Brown Cancer Center, Louisville, KY, USA; ${ }^{3}$ Yale University School of Medicine, New Haven, CT, USA; ${ }^{4}$ Providence Cancer Institute, Portland, OR, USA; ${ }^{5}$ University of Colorado, Aurora, CO, USA; ${ }^{6}$ Univ. of Florida Health Cancer Center, Orlando, FL, USA; ${ }^{7}$ NYU Langone Medical Center, New York, NY, USA; ${ }^{8}$ Atlantic Health System Cancer Care, Morristown, NJ, USA; ${ }^{9}$ Clinica Universitaria de Navarra, Pamplona, Spain; ${ }^{10}$ Addenbrooke's Hospital, Cambridge, UK; ${ }^{11}$ The Angeles Clinic and Research Institute, Los Angeles, CA, USA; ${ }^{12}$ Mount Sinai Comprehensive Cancer Center, Miami Beach, FL, USA; ${ }^{13}$ Szegedi Tudomanyegyetem Szent-Gyorgyi, Szeged, Hungary; ${ }^{14}$ The Royal Marsden NHS Foundation Trust, London, UK; ${ }^{15}$ lovance Biotherapeutics, San Carlos, CA, USA; ${ }^{16}$ University of Pittsburgh Medical Center, Pittsburgh, $P A$, USA

\subsection{6/LBA2019.18}

Background Treatment options are limited for patients with advanced melanoma who have progressed on checkpoint inhibitors and targeted therapies such as BRAF/MEK inhibitors (if BRAF-V600E mutated). Adoptive cell therapy utilizing tumor-infiltrating lymphocytes (TIL) has shown antitumor efficacy with durable responses in heavily pretreated melanoma patients. Safety and efficacy of lifileucel, a centrally manufactured cryopreserved autologous TIL therapy assessed by both investigator and an independent review committee (IRC), are presented.

Methods C-144-01 is a global Phase 2 open-label, multicenter study of the safety and efficacy of lifileucel in patients with unresectable metastatic melanoma. We report on Cohort $2(\mathrm{~N}$ = 66) patients with Stage IIIC/IV unresectable melanoma who received lifileucel. Tumors resected at local institutions were processed in central GMP facilities for TIL production in a 22-day process. Final TIL infusion product was cryopreserved and shipped to sites. Patients received one week of cyclophosphamide/fludarabine preconditioning lymphodepletion, a single lifileucel infusion, followed by up to 6 doses of IL-2. All responses were assessed by RECIST 1.1 .

Results 66 patients had the following baseline characteristics: 3.3 mean prior therapies (anti-PD1 100\%; anti-CTLA-4 80\%; BRAF/MEK inhibitor 23\%), relatively high tumor burden (106 $\mathrm{mm}$ mean target lesion sum of diameters), $44 \%$ with liver and/or brain lesions, median LDH $244 \mathrm{U} / \mathrm{L}$. Objective Response Rate (ORR) by investigator was 36.4\% (2 CR, 22 PR, 1 previously confirmed PR is now changed to SD) and Disease Control Rate (DCR) of $80.3 \%$. At a median follow up of 9.7 months, median Duration of Response (DOR) has not been reached. The adverse event profile was generally consistent with the underlying advanced disease and the profile of the lymphodepletion and IL-2 regimens.

The ORR per IRC was $34.8 \%$ (2 CR, 21 PR) and DCR was $72.7 \%$. At a median follow up of 6.9 months, the median IRC DOR has not been reached. Overall concordance rate of investigator and IRC read of response was 89.4\%. The concordance compares favorably with literature reports in a metastatic disease. ${ }^{1}$

Conclusions Lifileucel treatment resulted in a $36.4 \%$ ORR in heavily pretreated metastatic melanoma patients with high baseline disease burden who had received prior anti-PD1 and BRAF/MEK inhibitors, if tumor BRAF mutated. The high concordance of $89.4 \%$ between investigator and IRC confirms the original assessment of lifileucel efficacy in metastatic melanoma. $^{2}$

Acknowledgements The authors would like to thank the patients and their families for participation in the study.

The authors would also like to acknowledge the support and dedication of all investigators and site team members from all participating clinical trial institutions.

Trial Registration ClinicalTrials. gov Identifier: NCT02360579 Ethics Approval Ethics Approval This trial was approved by Western Institutional Review Board - IRB Tracking Number: 20160198.

\section{REFERENCES}

1. Ghiorghiu DC, et al. Comparison of central and site review of RECIST data in an open randomised phase II trial in advanced melanoma. 10.1594.ecr2009/C-075

2. Sarnaik $A$, et al. Safety and efficacy of cryopreserved autologous tumor infiltrating lymphocyte therapy (LN-144, lifileucel) in advanced metastatic melanoma patients who progressed on multiple prior therapies including anti-PD-1. I Clin Oncol 2019;37:2518-2518. 